\title{
Body Area Network based Health Monitoring of Critical Patients: a Brief Review
}

\author{
Amit Laddi ${ }^{1}$, Neelam R. Prakash ${ }^{2}$, Shashi Sharma ${ }^{1}$ and Amod Kumar ${ }^{1}$ \\ ${ }^{1}$ Central Scientific Instruments Organisation (CSIR-CSIO), Chandigarh, India \\ amitcsio@yahoo.com, *shashidu2@gmail.com, csioamod@yahoo.com \\ ${ }^{2}$ PEC University of Technology, Chandigarh, India \\ neelamrprakashpec@yahoo.com
}

\begin{abstract}
The paper discusses recent techniques for the detection of physical, chemical and biological signals along with their measurement and recording in case of children, elderly people and critical patients. Physical properties that can be sensed include temperature, pressure, vibration, sound level, weight, flow rate of gases and liquids, etc. The smart sensors which can be worn by the patient connect to the master hub (Central Computer) of the doctor sitting at a distance using wireless information and communication technology (ICT) network. The features of this technique include portability and non-invasive nature resulting in non-interference with the day to day activities of the patients. This technology is advantageous in the regions having limited resources and situations where continuous emergent diagnosis is required.
\end{abstract}

\section{KEYWORDS}

Physical, Chemical, Biological signals, Smart sensing, Body area network, Wireless

\section{INTRODUCTION}

One of the main challenges faced by the world in recent years has been the increase in the elderly population in developed countries. There is sharp increase in the rate of hospital admission for old age patients, therefore the governments across the world are dealing with problems related to unavailability of hospital beds as per the number of patients to be admitted. Not only the elderly and chronically ill, but also the families in which both parents have to work needs those systems which can provide high-quality care services for their babies and children. Therefore, various methods were developed having remote bio-monitoring system which bests suits for patients that are not as critical as ICU patients but require monitoring. Such systems can be used remotely in a home, an elderly institution or remote place to enable monitoring and detection of the vital signs of the patients, besides providing quality care [1].

The major components required for the development of Body area network based Condition Monitoring system are detecting the relevant quantities, monitoring and collecting the data, assessing and evaluating the information, information display devices, performing decisionmaking with alarm functions. Wireless sensor networks are responsible for sensing as well as transfer of information which should be fast, easy to install and maintain. 
International Journal of Instrumentation and Control Systems (IJICS) Vol.2, No.3, July 2012

\section{METHODOLOGY}

\subsection{Acquisition of Physiological Data}

Monitoring of physiological signals is done using two basic techniques viz. invasive and noninvasive.

Wearable and implantable body sensor network systems are the examples of invasive type of data acquisition techniques. Some papers focus on fall detection, posture detection and location tracking, while others make use of biological and environmental sensors to identify patient's health parameters. There is a significant research done for the development of tiny wireless sensor devices, which could be implanted in the patient body. The range of implantable biomedical devices increased significantly in the last few years due to evolution of micro-electro-mechanical systems (MEMS) technology, wireless communications, and digital electronics [2]. These advancements led to the development of small sized, low-cost, low power, multi-functional sensors which can communicate over short distances and apart from sensing, data processing, and communication can be performed by them.

The drawbacks of these monitoring devices consisting of leads and cables are that they usually restrict the mobility of patients along with the skin irritations or infections, etc. caused during implantation into patient body.

Therefore, to avoid such situations, non-invasive methods which consist of wearable sensors that touch the patient's body but do not require any operation for their attachments. The non-invasive methods included non-contacting sensors and connecting wires to the subjects where the biological signals are monitored using specially designed methods.

ECG can measured using capacitive coupling over clothes and PPG is measured non-intrusively during ordinary activities. Blood pressure is also estimated from ECG and PPG by calculating pulse arrival time (PAT). These methods can be applied to evaluate the health levels of subjects without intervening in their ordinary daily activities [3]. Some of the sensors do not require direct contact between instruments and bare skin. These methods were applied to the designs such as diagnostic chair for heart rate and blood pressure monitoring purposes comfortably.

These nonintrusive methods are used for simultaneous electrocardiogram, photoplethysmogram, and ballistocardiogram measurements. Ballistocardiogram (BCG) is the vital sign caused by the mechanical movement of the heart. Several type sensors have been developed to record BCG nonintrusively such as Phonocardiogram sensor, Polyvinylidene fluoride (PVDF) sensors, Multimodal optic sensors, Static-Charge-Sensitive Bed (SCSB) monitoring, Loadcell based method, etc. [4-9]. By simultaneously measuring signals, beat-to-beat heart rates could be monitored even when electrocardiograms were not recorded due to movement artifacts [10]. 


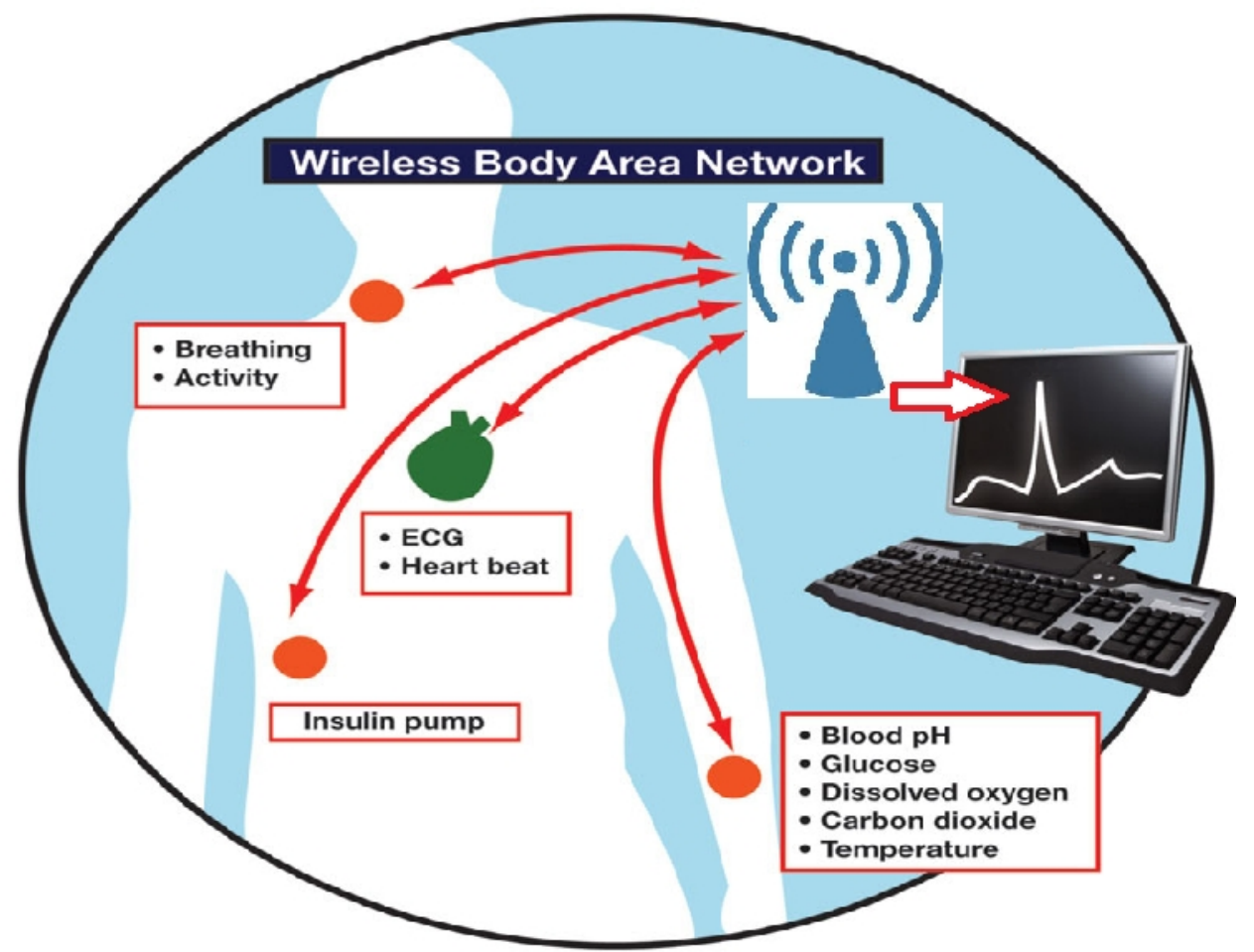

Figure 1. Measurement and transmission of Physiological data through Body area network

\subsection{Wireless Sensing Network}

As recent technological advances in integrated circuits, wireless communications, and physiological sensing allow miniature, lightweight, ultra-low power, intelligent monitoring devices, therefore a number of these devices can be integrated into a Wireless Body Area Network (WBAN). These types of smart sensors are integrated electronics that can perform one or more functions such as logic functions, two-way communication, decisions making, etc.

The wireless sensor networks consist of data acquisition devices along with data distribution network, monitored and controlled by a central computer. The basic design of communication network consists of nodes having computing power which can transmit and receive messages over wireless communication links. The basic network topologies include fully connected, mesh, star, ring, tree, bus or several interconnected subnets of different topologies. Networks are further classified as Local Area Networks (LAN), e.g. inside one building, or Wide Area Networks (WAN), e.g. between buildings, etc. The various types of wireless networks mostly used for making body area network are 802.11 Wireless Local Area Network, Bluetooth, etc. IEEE had ratified the IEEE 802.11 specification in 1997 as a standard for WLAN. Current versions of WiFi (802.11b) support transmission up to $11 \mathrm{Mbit} / \mathrm{s}$. The cost of installation of a WiFi router and receivers is within the budget and capability of common users. Bluetooth was initiated in 1998 and standardized by the IEEE as Wireless Personal Area Network (WPAN) specification IEEE 802.15. Bluetooth is a short range RF technology aimed at facilitating communication of electronic devices between each other and with the Internet, allowing for data synchronization that is transparent to the user. Almost all the common electronics gadgets are supported by this standard such as mobile devices, laptops, etc. Bluetooth uses the unlicensed $2.4 \mathrm{GHz}$ band and can transmit data up to $1 \mathrm{Mbit} / \mathrm{s}$, can penetrate solid non-metal barriers, and has a nominal range 
of $10 \mathrm{~m}$ that can be extended to $100 \mathrm{~m}$. A master station can service up to 7 simultaneous slave links. Forming a network of these networks, e.g. a piconet, can allow one master to service up to 200 slaves. Home RF was initiated in 1998 and has similar to Bluetooth for WPAN and has shared data/voice transmission. It interfaces with the Internet as well as the Public Switched Telephone Network and uses the $2.4 \mathrm{GHz}$ band with a range of $50 \mathrm{~m}$. A maximum of 127 nodes can be accommodated in a single network. IrDA is also a WPAN technology that has a shortrange, narrow-transmission-angle beam suitable for aiming and selective reception of signals.

An example of a medical WBAN used for patient monitoring is shown in Figure 1. Several sensors are placed in clothes, directly on the body or under the skin of a person and measure the temperature, blood pressure, heart rate, ECG, EEG, respiration rate, SpO2- levels etc. Next to sensing devices, the data is wirelessly transferred to central computer immediately (when a sensor notices a problem), which is connected to the doctor's end through internet who analyzes the data (Figure 2). One example is the monitoring of the glucose level in the blood of diabetics. If the sensor monitors a sudden drop of glucose, a signal can be sent to the central computer in order to provide the injection of insulin. In this way less time is wasted to provide the required medication to the patient.

Lots of work is going on towards the monitoring of physiological signals based on mobile telephony and internet. The challenges lie towards having low power consumption and light weight amidst other considerations such as bio vital signs detection and determination on a 24 by 7 basis by simply having a low power system located on the clothes of the patients which will enable the caregivers to respond to them in the fastest possible time [11-12].

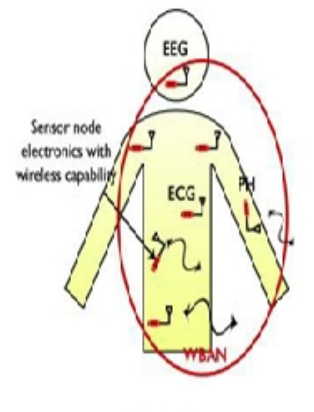

Patient
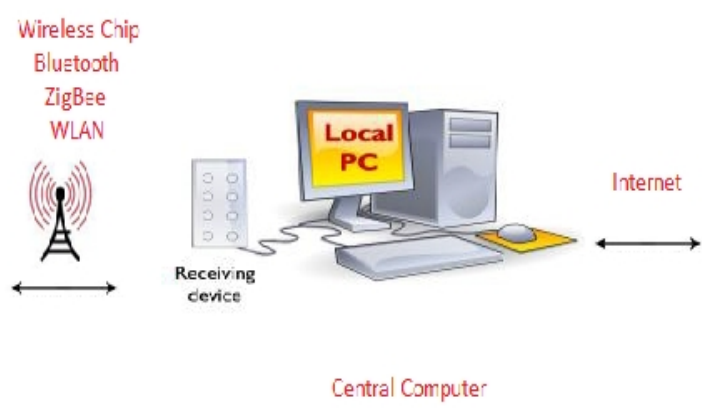

Central Computer

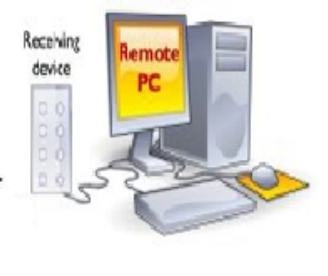

Master Control

(Doctor's End)

Figure 2. Remote Patient monitoring system

\section{CONCLUSIONS}

The advent of new enabling technologies for health monitoring are discussed briefly which can be used for long term measurement of biological signals and also, without interrupting patient's ordinary activities. These techniques are non-intrusive and meet the requirements for patient's health parameter monitoring. The non-intrusive methods for biological signal measurements have some disadvantages related to power consumption, consistency and range due to the fact the wireless body area networks usually prone to these types of problems. The authors have provided the brief information related to the physiological data acquisition in non- invasive mode, data processing and transfer to central hub along with alarm system to avoid any emergency situation. Further this data can be sent to the doctor's place to get the feedback or the required medication. This method can combine with various home equipments like beds, chairs, toilet seats and belts 
for measuring ECG, respiration, PPG and blood pressure, etc. The future studies are directed towards non-intrusive sensors diversification so that the measurement space can be extended for physiological parameters monitoring.

\section{ACKNOWLEDGEMENTS}

The authors would like to thank CSIR India for its funding and cooperation along with PEC University of technology, Chandigarh to give opportunity to perform this work.

\section{REFERENCES}

[1] E. Jovanov, A. Milenkovic, C. Otto, P. de Groen, "A wireless body area network of intelligent motion sensors for computer assisted physical rehabilitation”, J. Neuroeng. Rehabil., vol. 1, pp. 2-6, 2005.

[2] V. Leonov, P. Fiorini, S. Sedky, T. Torfs, C. van Hoof, "Thermoelectric MEMS generators as a power supply for a body area network", In Proceedings of the 13th International Conference on Solid-State Sensors, Actuators and Microsystems, pp. 291-294, Seoul, Korea, 5-9 June 2005.

[3] K. S. Park, "Nonintrusive measurement of biological signals for ubiquitous healthcare", 31st Annual International Conference of the IEEE EMBS, Minneapolis, Minnesota, USA, pp. 2-6 September, 2009.

[4] S. Tanaka, Y. Matsumoto, K. Wakimoto, "Unconstrained and non-invasive measurement of heartbeat and respiration periods using a phonocardiographic sensor", Med. Biol. Eng. Comput., vol. 40, no. 2, pp. 246-252, 1992.

[5] J. Jacobs, P. Embree, M. Glei, "Characterization of a novel heart and respiratory rate sensor," Conf. Proc. IEEE Eng. Med. Biol. Soc., vol. 3, pp. 2223-2226, 2004.

[6] W. B. Spillman, M. Mayer, J. Bennett, "A 'smart' bed for nonintrusive monitoring of patient physiological factors,” Meas. Sci. Tech., vol. 15, no. 8, pp. 1614-1620, Aug 2004.

[7] J. Alamets, E. Rauhala, E. Huupponena, A. Saastamoinen, A. Varri, A. Joutsen, J. Hasan, S.-L. Himanenc, "Automatic detection of spiking events in EMFi sheet during sleep," Med. Eng. Phys., vol. 28, pp. 267-275, 2006.

[8] J. Alihanka, K. Vaahtoranta, "A static charge sensitive bed. A new method for recording body movements during sleep,” Electroencephalogr. Clin. Neurophysiol., vol. 46, no. 6, pp. 731-734, Jun 1979.

[9] Y.J. Chee, J.M. Han, J.W. Youn, K.S. Park, "Air mattress sensor system with balancing tube for unconstrained measurement of respiration and heart beat movements, Physiol. Meas., vol. 26, pp. 413-422, 2005.

[10] H.J. Baek, G.S. Chung, K.K. Kim, K.S. Park , "A smart health monitoring chair for nonintrusive measurement of biological signals", IEEE Trans Inf Technol Biomed., 16(1):150-8, January 2012.

[11] J. M. Giachino, "Smart sensors," Sensors and actuators, vol. 10, pp. 239-248, 1986.

[12] S. Middelhoer and A.C. Hoogerwerf, "Smart sensors when and where," Sensors and Actuators, vol. 8, pp. 39-48, 1985.

\section{Authors}

\section{Biography of Amit Laddi}

Amit Laddi received the B.tech. (Hons.) degree in Electronics engineering from Kurukshetra University, India in 2004 and currently pursuing M.E. degree in Electronics Engineering from PEC University of Technology (2009-10 batch). He is currently working as a Scientist with Central Scientific Instruments Organisation (CSIR-CSIO), Chandigarh in Biomedical Instrumentation Division. His main research interests include machine vision and soft computing. 


\section{Biography of Neelam Rup Prakash}

Neelam Rup Prakash, Phd. did her B.E. in Electronics and Electrical Communications Engineering and M.E. degree in Electronics from Punjab University, Chandigarh. She has more than 22 years of experience in teaching. She is currently working as HOD in E\&EC, PEC University of Technology, Chandigarh. She has several publications in reputed journals. Her area of interest is wireless communications.

\section{Biography of Shashi Sharma}

Shashi Sharma received her B.E. and M.Tech. degrees from Institution of Engineers (India) and Punjab University Chandigarh in 1989 and 1999, respectively. She is a research engineer in Central Scientific Instruments Organisation, Chandigarh, and has more than 22 years experience in embedded systems and mixed circuit design. Her Research interests are Mixed-Signal Processing, DSP, Biomedical Signal Processing and Sensor Interface Electronics.

\section{Biography of Amod Kumar}

Amod Kumar, Ph.D. from IIT Delhi did his B.E. (Hons.) in Electrical and Electronics Engineering from Birla Institute of Technology and Science, Pilani (Raj.) in 1979 and M.E. degree in Electronics from Punjab University, Chandigarh. He has more than 32 years experience in Research and Development of different instruments in the areas of process control, environment, biomedical and prosthetics. He is currently working as Chief Scientist in Central Scientific Instruments Organisation, Chandigarh. He has 33 publications in reputed journals. He visited Germany under DAAD fellowship in 1987-88. His areas of interest are Embedded Systems, Digital Signal Processing and System Integration. 